\title{
ACTION OF NICOTINE ON THE ASCENDING RETICULAR ACTIVATING SYSTEM*
}

\author{
D. E. Knappt and E. F. Domino \\ Department of Pharmacology, University of Michigan, Ann Arbor, Michigan
}

\begin{abstract}
Summary-Synchronization of the electroencephalogram was produced by midpontine brainstem transection in the rabbit, cat and dog, and by high pontine transection in the monkey. After transection, i.v. injections of $10-20 \mu \mathrm{g} / \mathrm{kg}$ of nicotine produced EEG activation responses in all four species. The mechanism and site of action of the EEG activation effect of nicotine was explored in the dog. It has keen shown that peripheral nerve receptors are not essentially involved in nicotine induced EEG activation. Furthermore, the EEG activation effect of nicotine does not appear related to fluctuations in blood pressure, or to increases in circulating levels of epinephrine, norepinephrine, 5-hydroxytryptamine, or vasopressin. Studies in reserpine-pretreated dogs suggest nicotine does not owe its EEG activation effect to the release of central stores of catechol amines of 5-hydroxytryptamine. No evidence was obtained, however, which denies the possibilty that nicotine produced EEG activation by a cholinergic mechanism. It is tentatively concluded that a likely mechanism by which nicotine produces EEG activation is by mimicking, or possibly releasing, acetylcholine in the central nervous system. Because nicotine did not produce EEG activation in prepontine or postmammillary dogs, but altered electrical activity of the dog reticular slab, a ponto-mesencephalic site of action for the EEG activation effect of nicotine is proposed.

Although nicotine induced EEG activation does not appear to be mediated by an increase in circulating levels of vasopressin, attention is drawn to the possitilty that the release of vasopressin may be involved in the central nervous system pharmacology of nicotine, perhaps in regard to the development of acute tolerance to certain central actions of nicotine.

It is noted that in all four species studied, the $10-20 \mu \mathrm{g} / \mathrm{kg}$ EEG activation dose of nicotine corresponds to blood levels of nicotine which are commonly achieved in the habitual use of tobacco by man. Study of the central effects of nicotine analogs, particularly non-quarternary analogs, is suggested.
\end{abstract}

\section{INTRODUCTION}

Nicotine is known to have cardiovascular, respiratory, neuromuscular, and autonomic nervous system effects which do not involve participation of the central nervous system. Furthermore, by virtue of its stimulating action on a variety of peripheral nerve receptors, this alkaloid can initiate reflexly mediated changes in heart rate, blood pressure, and respiration. In spite of this multiplicity of pharmacological actions, some evidence has been obtained for central nervous system actions of nicotine on the spinal cord and on various vital centers of the medulla (see review by SILvETTE et al., 1962). Little has been presented, however, which supports the premise that nicotine, except in large doses, effects the higher centers. Sub-convulsive doses of nicotine have been reported to cause EEG activation in intact rabbits (LONGO et al., 1954; SilveSTRINI, 1958; STUMPF, 1959). Initial EEG activation also has been reported after the administration of convulsive doses of nicotine to high spinal or intact rabbits (VON BERGER and LONGO, 1953; LONGO et al., 1954; SilvestrinI, 1958; StumPF, 1959; and DUnLOP et al. 1960). Very recently FloRIS et al. (1962) showed that large doses of nicotine continue to produce EEG arousal or convulsions following mesencephalic transection in rabbits. The EEG convulsive activity

\footnotetext{
* This work was supported in part by a grant from the Tobacco Industry Research Committee.

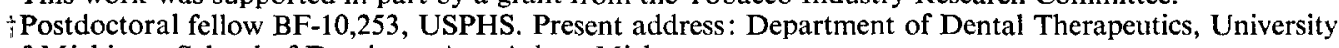
of Michigan, School of Dentistry, Ann Arbor, Mich.
} 
was more prolonged and steadier in the hippocampus than the cerebral cortex and appeared to originate in the hippocampus.

Although considerable attention generally has been directed toward the EEG convulsions evoked by nicotine, the mechanism of nicotine EEG activation has not been studied in detail. The present investigation was undertaken to determine if nicotine produced EEG activation by an action on the central nervous system, and to define the site and mechanism of this effect.

\section{General preparation of animals}

\section{METHODS}

Eighty-two brainstem transected animals were used for electroencephalographic studies. Adult animals of both sexes were used; they included ten albino rabbits, nine cats, fifty-four mongrel dogs, six Macaca cynomologus and three Macaca mulatta monkeys. The animals were prepared under diethyl ether anesthesia, or under local lidocaine anesthesia following immobilization with decamethonium. When indicated, respiration was maintained artificially with positive pressure ventilation. A femoral artery was cannulated for the recording of blood pressure with a mercury manometer, and a femoral or saphenous vein cannulated for the intravenous administration of drugs. Electrical activity, from sensorimotor cortex of rabbit, cat, and dog, and from motor cortex of the monkey, was recorded on a Grass electroencephalograph. Both monopolar and bipolar recordings were made using stainless steel needle electrodes. The elevated skin of the scalp was used as the indifferent site for monopolar recordings. When diethyl ether anesthesia was used, three hours were allowed for the elimination of this agent before the effect of drugs was tested. At the conclusion of each experiment the animal was terminated, a postmortem EEG record taken, and the calibration of the instrument repeated.

\section{Intercollicular, midpontine, pretrigeminal brainstem transection*}

To produce a stable pattern of EEG synchrony in the dog, midpontine transections were performed. Considerable variation in head size occurred in the mongrel dogs available for this study. It was noted, however, that the configuration of the interior of the dog skull presented numerous, prominent bony landmarks which could provide guides for the localization of the level of brainstem transection. Because stereotaxic orientation of the head depends upon extracranial structures, in particular the external auditory canals and the inferior border of the orbit, it was felt that the use of intracranial anatomical landmarks would yield more discretely localized transections in the dog.

After the dogs were anesthetized with diethyl ether, the calvarium was bared approximately $3 \mathrm{~cm}$ anterior to the posterior aspect of the external occipital protuberance and $1 \mathrm{~cm}$ left of the midline. A $1 \mathrm{~cm}$ circle of bone was removed from the exposed calvarium by trephination to reveal the dura mater overlying the cerebral cortex. The animal was given $1 \mathrm{mg} / \mathrm{kg}$ of decamethonium intravenously, and placed on artificial respiration. Parameters for positive pressure ventilation in the dog were $300 \mathrm{cc}$ room air $/ \mathrm{kg} / \mathrm{min}$ at 18 strokes/min, values found to maintain good color in the mucous membranes, brightred arterial blood, and stable blood pressure. The dura was slit and a thin, blunt edged spatula (Fig. 1A) introduced through the left hemisphere. The anterior aspect of the bony tentorium was located, and the spatula was gently lowered until the anterior aspect of

*This preparation will subsequently be referred to as the midpontine animal. 
the lateral dural portion of the tentorium cerebelli could be felt. The spatula was then directed just medial to the dural tentorium. The floccular fossa and internal acoustic meatus were located as definite depressions. The spatula was advanced slightly anterior. and the horizontal shelf for the passage of the trigeminal nerve was located. With the level of transection thus determined, the spatula was slowly raised and lowered across the width of the brainstem.

In order to achieve a complete transection, utilizing a unilateral cortical exposure so that the contralateral hemisphere would not be damaged, two additional spatulas (Fig. 1. $\mathrm{B}$ and $\mathrm{C}$ ) were employed in order to sever the neuronal connections of the right and superior aspects of the brainstem. Spatula B was gently lowered along the pathway already established by the first spatula. The second spatula then was employed to extend the transection to the opposite side, and to section all the remaining brainstem with the exception of the most superior connections of the opposite side.

Finally, the brainstem transection was completed with the more acutely angled spatula C. Inasmuch as this technique involves only minor surgical manipulation of the extracranial tissues, it was also possible to perform brainstem transections in the dog after immobilization with decamethonium and local lidocaine infiltration. In order to check the level and completeness of the transection, a postmortem examination of each brain was performed by midsagitally sectioning the head with a hacksaw and removing the brain after formalin fixation. Further deafferentation of the midpontine dog, to eliminate remaining peripheral nerve influences, was performed by bilateral section of the optic and oculomotor nerves and local instillation of 2 per cent lidocaine to the olfactory mucosia.

Brainstem transections were also performed in rabbits, cats, and monkeys with slight modifications of the above technique. Parameters for positive pressure ventilation in the rabbit, cat, and monkey were $400 \mathrm{cc}$ room air $/ \mathrm{kg} / \mathrm{min}$ at $24 \mathrm{strokes} / \mathrm{min}$. Minute volume values approximating these are outlined in the Handbook of Respiration (Altmas et al.. 1958).

\section{Intercollicular prepontine brainstem transection}

To explore the effect of a more rostral brainstem transection on EEG activation, particularly the activation produced by nicotine, prepontine rabbits, cats and dogs were prepared. The method was essentially the same as outlined for the midpontine animals except that the base of the dorsum sellae was used as the ventral bony landmark for the determination of the plane of section.

\section{Reticular slab preparation in the dog}

To isolate a portion of the brainstem from ascending and descending influences, reticular slabs were prepared by an intercollicular, midpontine, pretrigeminal transection caudally. and an intercollicular, prepontine transection rostrally. Electrical activity, as recorded by a stainless steel macroelectrode in such brainstem slabs, rapidly deteriorated and no satisfactory records could be obtained. On the assumption that the rapid loss of elcctrical activity was related to impaired blood supply, subsequent slabs were prepared by an intercollicular, midpontine, pretrigeminal section caudally, and a precollicular, postmammillary section rostrally. The more rostral section was started approximately $5 \mathrm{~mm}$ anterior to the bony tentorium and directed to the anterior aspect of the dorsum sellae. A stainless steel wire macroelectrode, insulated with epoxylite to within $1 \mathrm{~mm}$ of the tip, was inserted into the slab and fastened to the cranial bone by means of a thermoplastic dental impression 
compound. At the end of each experiment, electrode placement in the slab was confirmed. A lesion was produced at the electrode tip by passing a high intensity coagulating current from a Wappler electrosurgical unit for $5 \mathrm{sec}$. After formalin fixation, the reticular slab was removed, and the $2-3 \mathrm{~mm}$ lesion located macroscopically by serially slicing the tissue with a scalpel.

\section{Catechol amine and 5-hydroxytryptamine assays}

All dogs used for the assays, including normal controls, were anesthetized with diethyl ether and transected at a midpontine level. The animals were then sacrificed by exsanguination through the femoral artery, the brain quickly removed, and the brainstem (medulla, pons, mesencephalon, diencephalon) isolated and weighed. The brainstem was immediately homogenized in a Dounce ball-type homogenizer (Blaessig Glass Co.) with $20 \mathrm{ml} \mathrm{of} 0.4 \mathrm{~N}$ perchloric acid. Additional $0.4 \mathrm{~N}$ perchloric acid was added to make a total of $40 \mathrm{ml}$ of homogenate. The resultant mixture was rehomogenized. Two $8 \mathrm{ml} \mathrm{samples,} \mathrm{each} \mathrm{represent-}$ ing $1 / 5$ of the total sample, were transferred to celluloid centrifuge tubes, one sample for catechol amine assay, the other for the assay of 5-hydroxytryptamine.

The method of catecholamine assay was the spectrophotofluorometric method described by Bertler, CARlsSon, and Rosengren (1958) as modified by MOJRE and Brody (1961).

A modification of the 5-hydroxytryptamine assay method of BogDANSKI et al. (1956) was utilized, as suggested by Dr. T. M. Brody (personal communication) in order to use the perchloric acid brainstem homogenate described above. An $8 \mathrm{ml}$ sample of homogenate was centrifuged, and the supernatant liquid collected. The residue was resuspended in an additional $4 \mathrm{ml}$ of $\cdot 4 \mathrm{~N}$ perchloric acid. The suspension was again centrifuged, and the supernatant liquids pooled. Five $\mathrm{M} \mathrm{K}_{2} \mathrm{CO}_{3}$ was added to the pooled supernatants to $\mathrm{pH} 4$, and the resulting suspension was then centrifuged to remove the precipitated potassium perchlorate. 5-Hydroxytryptamine was extracted from the perchlorate-free supernatant by adding $\mathrm{Na}_{2} \mathrm{CO}_{3}$ to $\mathrm{pH} 10$ and extracting in n-butanol. The 5-hydroxytryptamine was subsequently extracted from the n-butanol into $0 \cdot 1 \mathrm{~N} \mathrm{HCl}$. The acid extract was then used for the fluorometric determination of 5-hydroxytryptamine, as outlined by BoGDANSKI et al. (1956).

\section{RESULTS}

Transection of the brainstem in six dogs, at an intercollicular, prebulbar level (at the level of the internal acoustic meatus), produced no evidence of slow waves or spindles in the EEG. When transection was subsequently performed slightly more rostrally in the same dogs, at an intercollicular, midpontine pretrigeminal level, a marked alteration of the EEG pattern was seen. The most prominent change was the appearance of $10-14 \mathrm{cps}$ spindle bursts, which occurred at a mean frequency \pm SD of $13 \cdot 1 \pm 2 \cdot 98 / \mathrm{min}$. Between the EEG spindles there appeared occasional slow waves and bursts of low voltage, fast wave activity. No evidence of spontaneous EEG activation was seen in these or in subsequently prepared midpontine dogs.

No midpontine dog was studied longer than $18 \mathrm{hr}$, for intracranial bleeding and cerebral edema proved to be major problems in the maintenance of EEG activity in brainstem transected dogs. Especially after large or repeated pressor responses, EEG activity generally deteriorated to delta waves and finally to an isoelectric state. These EEG changes were 


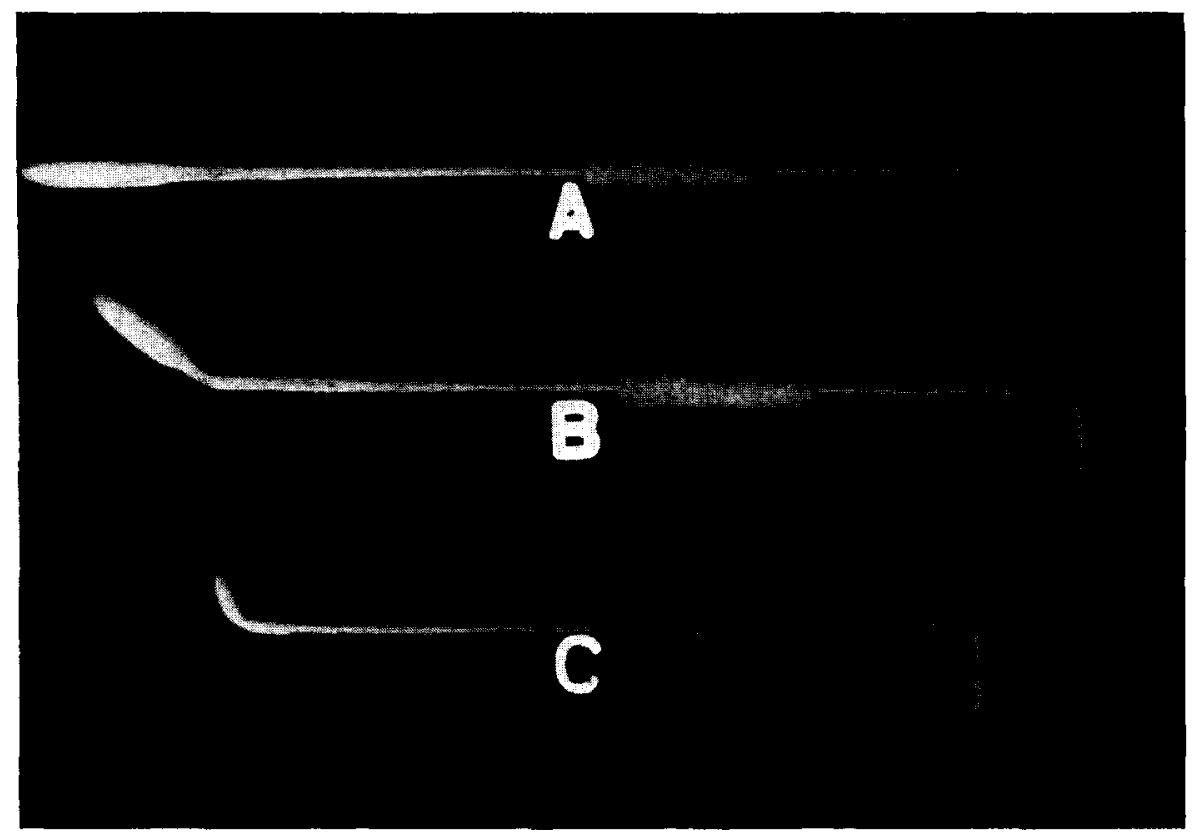

FiG. 1. Instruments used for brainstem transection. Instrument $\mathrm{A}$ was used for detecting bony landmarks and establishing plane of section. Instruments B and C were used for the completion of transection.

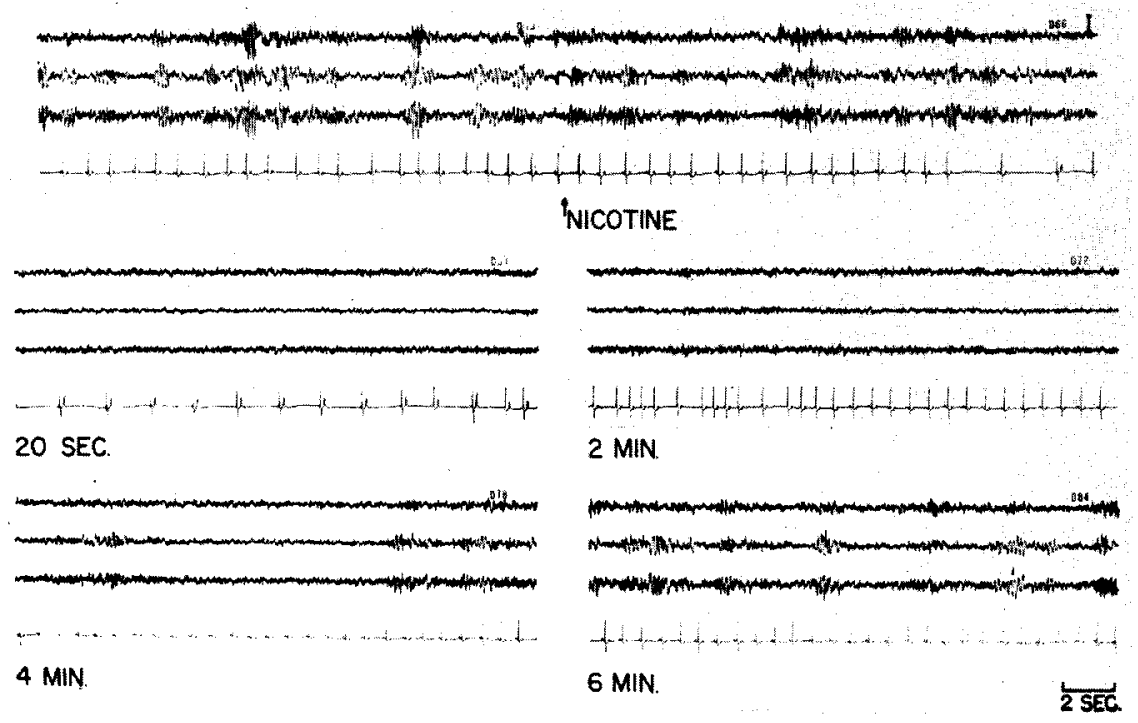

FIG. 2. Nicotine induced EEG activation in the midpontine dog. Approximately 20 sec after the i.v. administration of $10 \mu \mathrm{g} / \mathrm{kg}$ nicotine, EEG spindles disappear and low voltage, fast frequency waves predominate. Four minutes after injection spindling resumes. Six min after injection, spindles are again prominent. Channels $1-4$ are respectively monopolar right sensorimotor cortex, monopolar left sensorimotor cortex, bipolar right to left sensorimotor cortex, and lead II EKG. Calibration bar: $100 \mu \mathrm{V}$. 


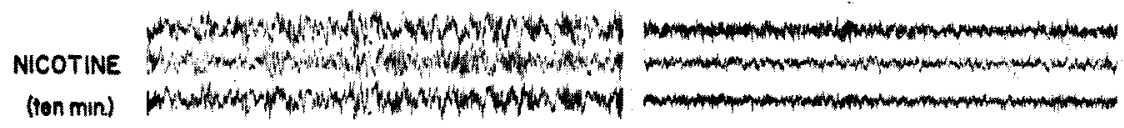

FIG. 3. Nicotine specificity in the midpontine dog. As seen in the top panels, $20 \mu \mathrm{g} / \mathrm{kg}$ of the quarternary ganglionic stimulant, DMPP, did not produce EEG activation. In contrast, the EEG activation effect of $10 \mu \mathrm{g} / \mathrm{kg}$ nicotine, given $10 \mathrm{~min}$ later, is clearly illustrated in the lower panels. Left hand panels control, right hand panels 1 min after injection. Channels and calibration as in Fig. 2.

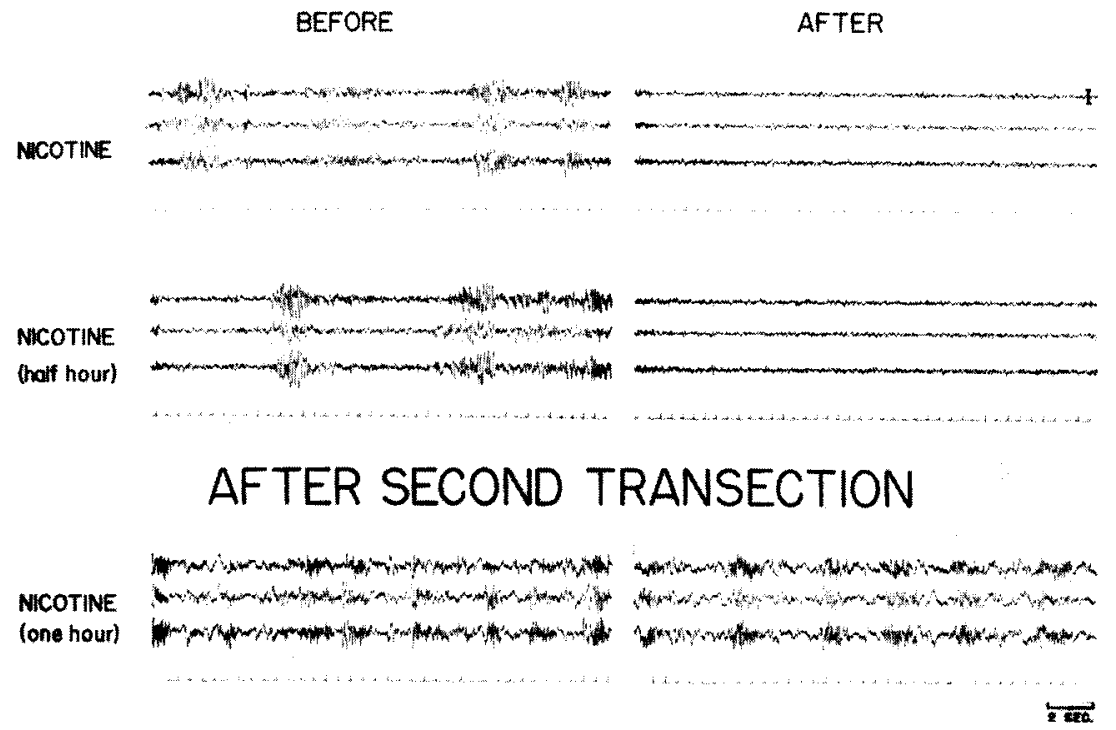

FIG. 4. The effect of level of transection on nicotine induced EEG activation. The top panels illustrate nicotine induced EEG activation in the midpontine dog. One-half hr later, $10 \mu \mathrm{g} / \mathrm{kg}$ of nicotine again produced EEG activation, as seen in the middle panels. After prepontine section, in spite of allowing an hour between injections, $20 \mu \mathrm{g} / \mathrm{kg}$ of nicotine did not produce an EEG activation response, as illustrated in the lower panels. Note the enhancement of slow waves and increased frequency of spindling after prepontine section. Left hand panels control, right hand panels 1 min after injection. Channels and calibration as in Fig. 2. 


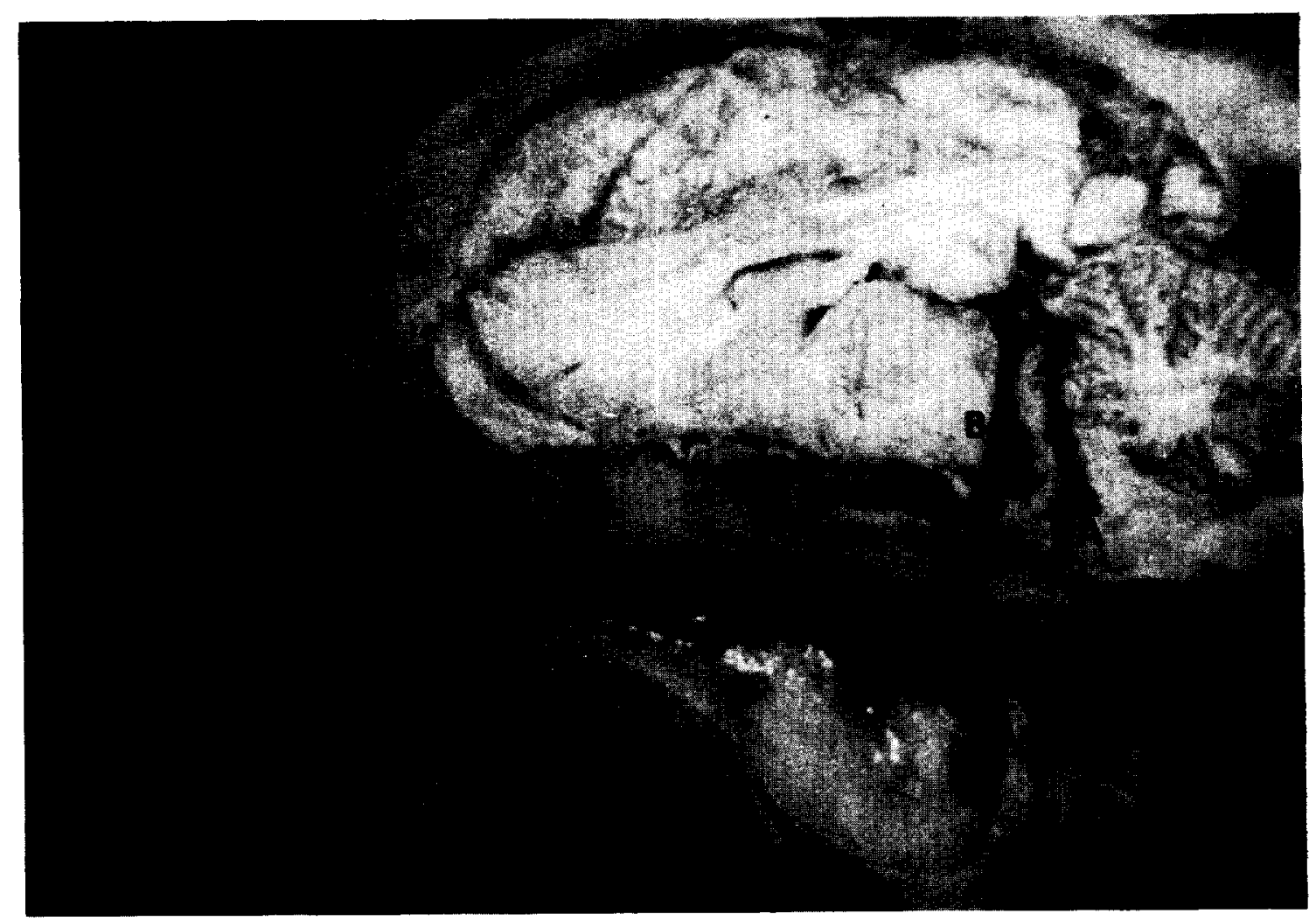

Fig. 5. Midsagittal section of a brainstem transected dog (Mag approx. $1 \frac{1}{2} \mathrm{x}$ ) Section A-Midpontine-Nicotine positive Section B-Prepontine-Nicotine negative 


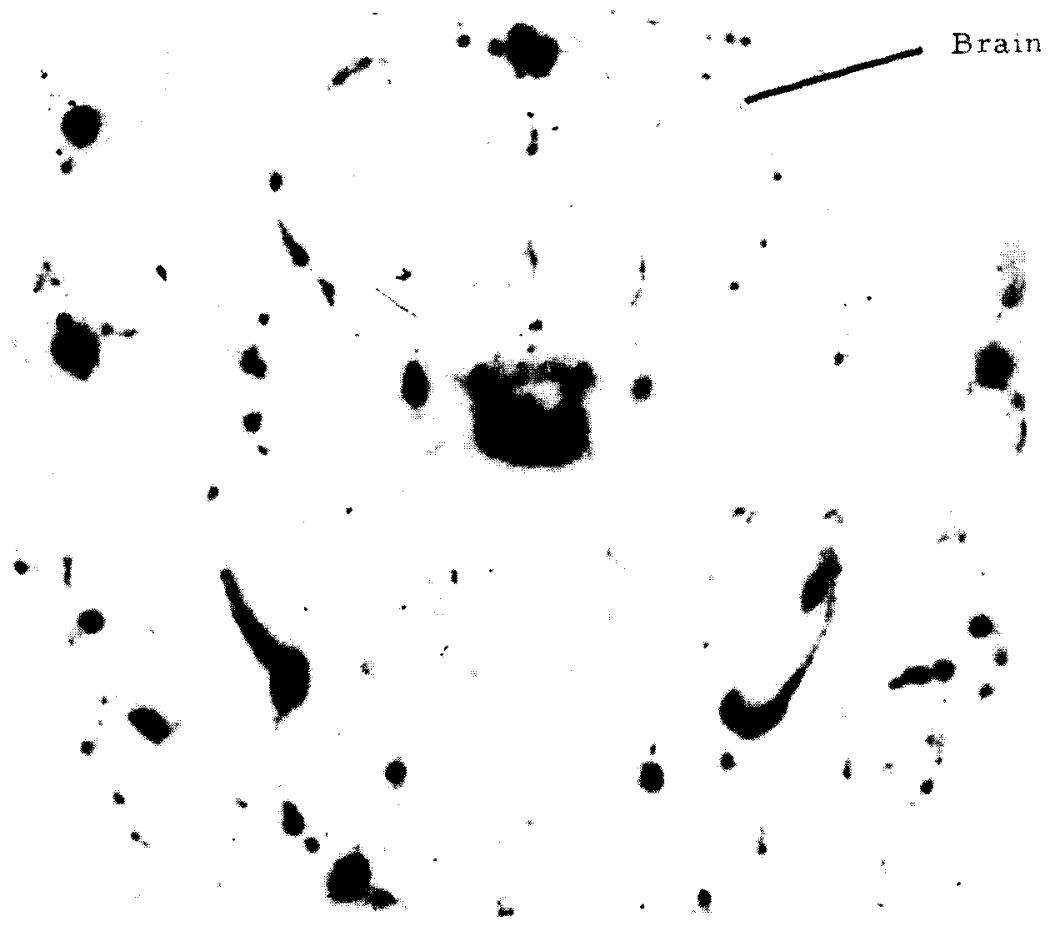

FIG. 8. Autoradiogram obtained from a coronal section of the frozen rat head at 5 min after i.v. injection of $\left.\right|^{131}$-radio-iodinated serum albumin (RISA, Abbott). The black punctate spots represent radioactivity in the larger blood vessels.

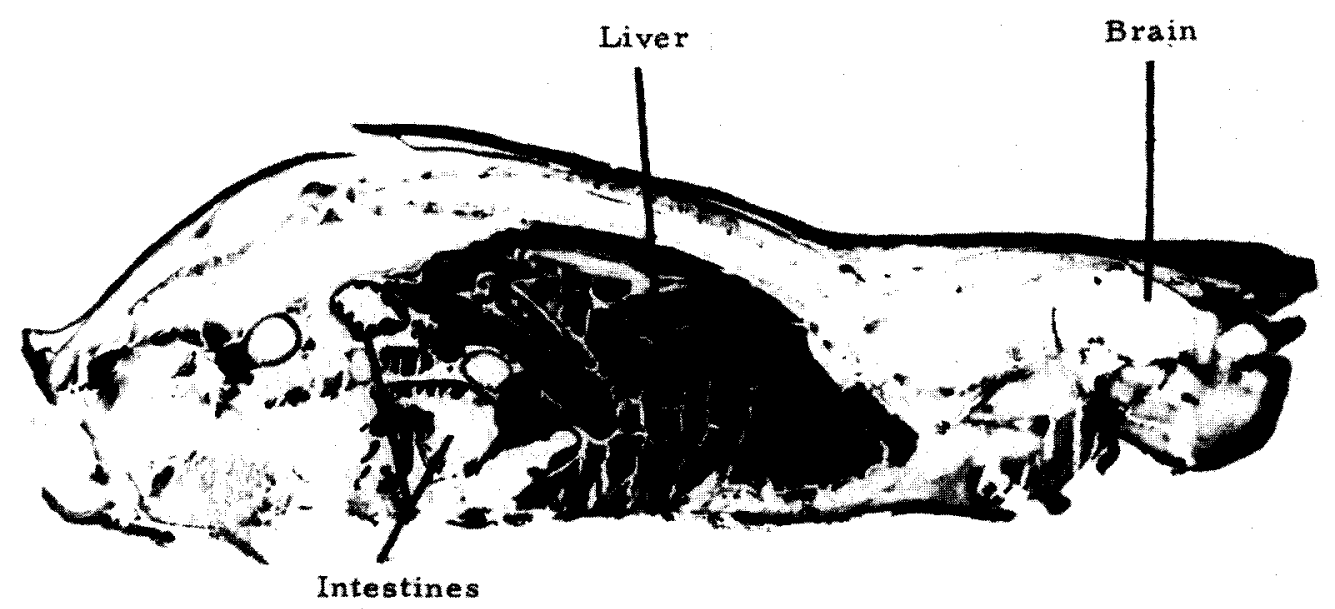

FlG. 9. Autoradiogram showing the distribution of $\mathrm{S}^{35}$ in a mouse 5 min after i.v. injection of $\mathrm{S}^{35}$-sodium sulfate. 